\title{
On Bicomplex Representation Methods and Applications of Matrices over Quaternionic Division Algebra*
}

\author{
Junliang Wu, Pingping Zhang \\ College of Mathematics \& Statistics, Chongqing University, Chongqing, China \\ E-mail: jlwu678@tom.com, zhpp04010248@163.com \\ Received January 6, 2011; revised January 20, 2011; accepted January 25, 2011
}

\begin{abstract}
In this paper, a series of bicomplex representation methods of quaternion division algebra is introduced. We present a new multiplication concept of quaternion matrices, a new determinant concept, a new inverse concept of quaternion matrix and a new similar matrix concept. Under the new concept system, many quaternion algebra problems can be transformed into complex algebra problems to express and study. These concepts can perfect the theory of [J.L. Wu, A new representation theory and some methods on quaternion division algebra, JP Journal of Algebra, 2009, 14(2): 121-140] and unify the complex algebra and quaternion division algebra.
\end{abstract}

Keywords: Quaternion Determinant, Product of Quaternion Matrix, Inverse of Quaternion Matrix, Similar Quaternion Matrix, Application, Solution

\section{Introduction}

In recent years, the algebra problems over quaternion division algebra have drawn the attention of mathematics and physics researchers [1-12]. Quaternion algebra theory is getting more and more important. In many fields of applied science, such as physics, figure and pattern recognition, spacecraft attitude control, 3-D animation, people start to make use of quaternion algebra theory to solve some actual problems. Therefore, it encourages people to do further research [13-17] on quaternion algebra theory and its applications.

The main obstacle in the study of quaternion algebra is the non-commutative multiplication of quaternion. Many important conclusions over real and complex fields are different from ones over quaternion division algebra, such as determinant, the trace of matrix multiplication and solutions of quaternion equation. From the conclusions on quaternion division algebra, we find it to lack for general concepts, such as the definition of quaternion matrix determinant. There are different definitions which are given in $[1,3,4,6,11,18]$ since Dieudonne firstly introduced the quaternion determinant in 1943. In addition, the inverse of quaternion matrix has not been well de-

*This work was supported by National Natural Science Foundation of China (No. 70872123) and Science Research Foundation of Chongqing city of China (09-03-029). fined so far, because it depends on other algebra concepts. In the study of quaternion division algebra, people always expect to get some relations between quaternion division algebra and real algebra or complex algebra. However, some conclusions on real or complex fields are correct but not on quaternion division algebra. It makes us to consider establishing other algebra concept system over quaternion division algebra to unify the complex algebra and quaternion division algebra.

Recently, Wu in [19] used real representation methods to express quaternion matrices and established some new concepts over quaternion division algebra. From these definitions, we can see that they can convert quaternion division algebra problems into real algebra problems to reduce the complexity and abstraction which exist in all kinds of definitions given in $[1,3,6,10,11,20]$. However, as $\mathrm{Wu}$ in [19] mentioned, these concept system is not suitable for complex algebra.

In this paper, based on the bicomplex form of quaternion matrix, we present some new concepts to quaternion division algebra. These new concepts can perfect the theory of Wu in [19] and unify the complex algebra and quaternion division algebra.

This paper is organized as follows. In Section 2, we introduce a complex representation method of quaternion matrices and explore the relation between quaternion matrices and complex matrices. In Section 3, we present 
a series of new concepts over quaternion division algebra and study their properties. In section 4, we establish some important theorems to illustrate the applications and effectiveness of the new concept system.

Let $C$ denote the complex field, $H$ denote the quaternion set, $\boldsymbol{C}^{m \times n}$ denote the set of $m \times n$ complex matrices, $\boldsymbol{H}^{m \times n}$ denote the set of $m \times n$ quaternion matrices and $\boldsymbol{A}^{T}$ denote the transpose matrix of $\boldsymbol{A}$.

\section{The Bicomplex Representation Methods of Quaternion Matrices and the Relation between Quaternion Matrices and Complex Matrices}

For any quaternion matrix $\boldsymbol{A} \in \boldsymbol{H}^{m \times n}, \boldsymbol{A}$ can be uniquely represented as

$$
\boldsymbol{A}=\boldsymbol{A}_{0}+\boldsymbol{A}_{1} j
$$

where $\boldsymbol{A}_{s} \in \boldsymbol{C}^{m \times n}(s=0,1), \quad \boldsymbol{A}_{1} j$ means to multiply each entries of $A_{1}$ by $j$ from right hand side.

For above reasons, we can establish a mapping relation between quaternion matrices and complex matrices as follows:s

$$
f: \boldsymbol{A} \in \boldsymbol{H}^{m \times n} \mid \rightarrow\left(\boldsymbol{A}_{0}, \boldsymbol{A}_{1}\right),
$$

where $\boldsymbol{A}_{s} \in \boldsymbol{C}^{m \times n}(s=0,1)$.

The set of $m \times n$ quaternion matrices is written as $\boldsymbol{A}$ and the set of image of $\boldsymbol{A}$ is written as $\boldsymbol{A}_{i m g}$.

Theorem 2.1. Let $f: \boldsymbol{A} \in \boldsymbol{H}^{m \times n} \mid \rightarrow\left(\boldsymbol{A}_{0}, \boldsymbol{A}_{1}\right)$, $\left(\boldsymbol{A}_{s} \in \boldsymbol{C}^{m \times n}(s=0,1)\right)$. Then the mapping $f$ is a bijective mapping from $\boldsymbol{A}$ to $\boldsymbol{A}_{i m g}$.

Proof. For any entry $\left(A_{0}, A_{1}\right)$ in $\boldsymbol{A}_{i m g}$, there exists the corresponding quaternion matrix $\boldsymbol{A}=\boldsymbol{A}_{0}+\boldsymbol{A}_{1} j$ in $\boldsymbol{A}$, therefore $f$ is a surjection from $\boldsymbol{A}$ to $\boldsymbol{A}_{\text {img }}$. Simultaneously, since any quaternion matrix in $\boldsymbol{A}$ can be uniquely represented as the form (2.1), so $f$ is an injection from $\boldsymbol{A}$ to $\boldsymbol{A}_{i m g}$. Thus $f$ is a bijective mapping from $\boldsymbol{A}$ to $\boldsymbol{A}_{\text {img }}$.

The proof is complete.

Theorem 2.2. Bijection $f: \boldsymbol{A}|\leftrightarrow|\left(\boldsymbol{A}_{0}, \boldsymbol{A}_{1}\right)$, $A_{s} \in C^{m \times n}(s=0,1)$ is an isomorphism mapping from $\boldsymbol{A}$ to $\boldsymbol{A}_{\text {img }}$.

By the concept of isomorphism mapping, this theorem is easy to prove and we omit it here.

We shall mention that Theorem 2.2 is the foundation of this article, because isomorphism vector spaces have the same properties.

\section{The Bicomplex Matrix Concept System over Quaternion Division Algebra}

According to the complex representation of quaternion matrices above, a series of new definitions of quaternion division algebra which are helpful to discuss the algebra problems on quaternion division algebra can be given as follows.

Definition 3.1. The matrix $\tilde{\boldsymbol{E}}=\boldsymbol{E}+\boldsymbol{E} \boldsymbol{j}$ is said to be a $n \times n$ unit quaternion matrix if $E$ is a $n \times n$ unit matrix over complex field. In particular, if $n=1$, then $\tilde{\boldsymbol{E}}=\boldsymbol{E}+\boldsymbol{E} \boldsymbol{j}=\mathbf{1}+\mathbf{1} j$ is said to be a unit quaternion written as $a_{u}$.

Definition 3.2. Let and $\boldsymbol{B}=\boldsymbol{B}_{0}+\boldsymbol{B}_{1} j \in \boldsymbol{H}^{n \times t}$ be given. The operator $\boldsymbol{A} * \boldsymbol{B}=\boldsymbol{A}_{0} \boldsymbol{B}_{0}+\boldsymbol{A}_{1} \boldsymbol{B}_{1} j$ (where $\boldsymbol{A}_{0} \boldsymbol{B}_{0}, \boldsymbol{A}_{1} \boldsymbol{B}_{1}$ are both the multiplications of complex matrices) is called the *-product of quaternion matrices $A$ and $B$. In particular, if $m=n=t=1$, then we can derive the *-product of quaternions.

Note: when $\boldsymbol{A} \in \boldsymbol{C}^{m \times n}, \boldsymbol{B} \in \boldsymbol{C}^{n \times t}$, then $\boldsymbol{A} * \boldsymbol{B}=\boldsymbol{A} \boldsymbol{B}$.

Under the Definition 3.1 and Definition 3.2, we give some relative properties.

For any matrix $\boldsymbol{A}, \boldsymbol{B} \in \boldsymbol{H}^{n \times n}$, we have:

1) $\tilde{\boldsymbol{E}} * \boldsymbol{A}=\boldsymbol{A} * \tilde{\boldsymbol{E}}=\boldsymbol{A}$, where $\tilde{\boldsymbol{E}}$ is a $n \times n$ unit quaternion matrix;

2) $\boldsymbol{A}+\boldsymbol{B}=\boldsymbol{B}+\boldsymbol{A}$

3) $(\boldsymbol{A}+\boldsymbol{B}) * \boldsymbol{C}=\boldsymbol{A} * \boldsymbol{C}+\boldsymbol{B} * \boldsymbol{C}$;

4) $(\boldsymbol{A} * \boldsymbol{B})^{T}=\boldsymbol{B}^{T} * \boldsymbol{A}^{T}$;

5) $\operatorname{Tr}(\boldsymbol{A} * \boldsymbol{B})=\operatorname{Tr}(\boldsymbol{B} * \boldsymbol{A})$.

Similarly, we establish a new definition as follows. Definition 3.3. Let $\boldsymbol{X} \in \boldsymbol{H}^{n \times 1}$ and $a \in \boldsymbol{H}$ be given. Then $a * \boldsymbol{X}=\boldsymbol{X} * a=a_{0} \boldsymbol{X}_{0}+a_{1} \boldsymbol{X}_{1} j$ is called the *-product of quaternion and quaternion vector, where $\boldsymbol{X}=\boldsymbol{X}_{0}+\boldsymbol{X}_{1} j$, $\boldsymbol{X}_{0} \in \boldsymbol{C}^{n \times 1}, \boldsymbol{X}_{1} \in \boldsymbol{C}^{n \times 1}, \quad a=a_{0}+a_{1} j, a_{0} \in C, a_{1} \in C$.

Now, we introduce the following concept to quaternion division algebra.

Definition 3.4. For any quaternion matrix $\boldsymbol{A} \in \boldsymbol{H}^{n \times n}$ $\left(\boldsymbol{A}=\boldsymbol{A}_{0}+\boldsymbol{A}_{1} j\right), \quad\|\boldsymbol{A}\|=\left|\boldsymbol{A}_{0}\right|+\left|\boldsymbol{A}_{1}\right| j$ is said to be the determinant of $\boldsymbol{A}$, where $|$.$| is the determinant of a com-$ plex matrix.

Note: when $\boldsymbol{A} \in \boldsymbol{C}^{n \times n}$, then $\|\boldsymbol{A}\|=|\boldsymbol{A}|$.

The Definition 3.3 is reasonable. First of all, the result of a quaternion matrix determinant under Definition3.4 is a quaternion. Secondly, from Definition 3.4 we can see that it can convert the determinant of a quaternion matrix into that of complex matrices to reduce the complexity and abstraction. Finally, the new determinant has the same fundamental properties as that over complex field. That is, if $\boldsymbol{A}$ is a $n \times n$ quaternion matrix and $i \neq j$, then we have

1) $\|\boldsymbol{A}\|=\left\|\boldsymbol{A}^{T}\right\|$.

2) If quaternion matrix $\boldsymbol{B}$ is obtained from quaternion matrix $\boldsymbol{A}$ by interchanging two rows (or columns) of $\boldsymbol{A}$, then $\|\boldsymbol{B}\|=-\|\boldsymbol{A}\|$. 
3) If quaternion matrix $\boldsymbol{A}$ has a zero row (or column), then $\|\boldsymbol{A}\|=\left\|\boldsymbol{A}^{T}\right\|=0$.

4) $\|k * \boldsymbol{A}\|=k^{* n}\|\boldsymbol{A}\|$, where $k^{* n}=\underbrace{k * k * \cdots * k}_{n}, \quad k \in \boldsymbol{H}$.

5) If the $j$ th row (column) of quaternion matrix $A$ equal a multiple of the ith row (column) of the matrix, then $\|A\|=0$.

6) Suppose that $\boldsymbol{A}, \boldsymbol{B}$ and $\boldsymbol{C}$ are all $n \times n$ quaternion matrices. If all rows of $\boldsymbol{B}$ and $\boldsymbol{C}$ both equal the corresponding to rows (columns) of $\boldsymbol{A}$ except that the $i$ th row (column) of $\boldsymbol{A}$ equal the sum of the ith of $\boldsymbol{B}$ and $\boldsymbol{C}$, then $\|\boldsymbol{A}\|=\|\boldsymbol{B}\|+\|\boldsymbol{C}\|$.

7) If quaternion matrix $\boldsymbol{B}$ is the $n \times n$ matrix resulting from adding a multiple of the ith row (or column) of matrix $\boldsymbol{A}$ to the $j$ th row (or column) of matrix $\boldsymbol{A}$, then $\|\boldsymbol{B}\|=\|\boldsymbol{A}\|$.

8) Let $\boldsymbol{A}$ and $\boldsymbol{B}$ be $n \times n$ quaternion matrices respectively. We have $\|\boldsymbol{A} * \boldsymbol{B}\|=\|\boldsymbol{A}\| *\|\boldsymbol{B}\|$.

Up to now, people still treat the inverse matrix concept of quaternion matrix as complex matrix, that is, if quaternion matrix $\boldsymbol{A}$ satisfies $\boldsymbol{A}^{-1} \boldsymbol{A}=\boldsymbol{E}$ (where $\boldsymbol{E}$ is a real unit matrix), then people think that quaternion matrix $\boldsymbol{A}$ exists its inverse matrix $\boldsymbol{A}^{-1}$. However, people pointedly ignore two questions. An issue is how to define the product of quaternion matrices $\boldsymbol{A}^{-1}$ and $\boldsymbol{A}$. The other one is how to make a calculation of $\boldsymbol{A}^{-1}$.

It indicates that the terminology of inverse matrix does not have a clear definition in quaternion algebra theory.

In the following, we shall give a new definition and specific computational method for the inverse of quaternion matrix.

Definition 3.5. Let $\boldsymbol{A}=\boldsymbol{A}_{0}+\boldsymbol{A}_{1} \boldsymbol{j} \in \boldsymbol{H}^{n \times n}$ be given (where $A_{0}, A_{1}$ both are complex matrices). If the inverse matrices of $A_{0}$ and $A_{1}^{-1}$ both exist, then quaternion matrix $\boldsymbol{A}$ is said to be invertible and the inverse matrix is written as $\boldsymbol{A}^{-}=\boldsymbol{A}_{0}^{-1}+\boldsymbol{A}_{1}^{-1} \boldsymbol{j}$, where $\boldsymbol{A}_{0}^{-1}, \boldsymbol{A}_{1}^{-1}$ denote the inverse of complex matrices $\boldsymbol{A}_{0}, A_{1}$ respectively.

Note: when $\boldsymbol{A} \in \boldsymbol{C}^{n \times n}$, then $\boldsymbol{A}^{-}=\boldsymbol{A}^{-1}$.

The inverse of quaternion matrix under the new definition has the same fundamental properties as those under the traditional algebra system. It is easy to show the following facts by the new concept, namely, if a quaternion matrix $\boldsymbol{A}$ is invertible, then we have:

1) $\left(\boldsymbol{A}^{-}\right)^{-}=\boldsymbol{A}$.

2) $\left(A^{-}\right)^{k}=\left(A^{k}\right)^{-}=\left(A_{0}^{-1}\right)^{k}+\left(A_{1}^{-1}\right)^{k} j$, where

$A^{k}=A * A * \cdots * A$ is product of $k \boldsymbol{A}$ which is defined in Definition 3.2.

3) If $A_{1}, A_{2}, \cdots, A_{m}$ are all invertible quaternion matrices, then

$$
\left(A_{1} * A_{2} * \cdots * A_{m}\right)^{-}=A_{m}^{-} * A_{m-1}^{-} * \cdots * A_{1}^{-} .
$$

Obviously, by the new definition of inverse of quaternion matrix above, people can determine easily whether the inverse matrix of quaternion matrix exists or not and calculate the inverse matrix if possible.

Under the definition of inverse of quaternion matrix above, a new concept of similar quaternion matrices can be given as follows:

Definition 3.6. Let $\boldsymbol{A}, \boldsymbol{B} \in \boldsymbol{H}^{n \times n}$, if there exists an invertible quaternion matrix $\boldsymbol{P}$ such that $\boldsymbol{A}=\boldsymbol{P}^{-} * \boldsymbol{B} * \boldsymbol{P}$, then $\boldsymbol{A}$ and $\boldsymbol{B}$ are said to be similar quaternion matrices written as $\boldsymbol{A} \sim \boldsymbol{B}$.

Note: when $\boldsymbol{A}, \boldsymbol{B} \in \boldsymbol{C}^{n \times n}, \boldsymbol{A}=\boldsymbol{P}^{-} * \boldsymbol{B} * \boldsymbol{P}$ is equivalent to $\boldsymbol{A}=\boldsymbol{P}_{0}^{-1} \boldsymbol{B} \boldsymbol{P}_{0}$, where $\boldsymbol{P}=\boldsymbol{P}_{0}+\boldsymbol{P}_{1} \boldsymbol{j}, \boldsymbol{P}_{0}, \boldsymbol{P}_{1} \in \boldsymbol{C}^{n \times n}$.

For similar quaternion matrices, we will deduce many important properties in the next section.

\section{Some Applications of the Bicomplex Matrix Concept System}

In this section, we establish some important theorems to illustrate the applications and effectiveness of the new concept system for the research of quaternion division algebra. The eigenvalue is an important issue in quaternion division algebra theory, so under the new concept system, we will study firstly the eigenvalues of quaternion matrix and the relation between eigenvalues of similar quaternion matrices in detail.

Before showing the application, we'll introduce firstly some concepts associated with eigenvalue.

Definition 4.1. For any matrix $\boldsymbol{A}=\left(a_{i j}\right) \in \boldsymbol{H}^{n \times n}$, if there exists nonzero quaternion vector $\boldsymbol{X} \in \boldsymbol{H}^{n \times 1}$ and a quaternion $\lambda=\lambda_{0}+\lambda_{1} j$ (where $\lambda_{0}, \lambda_{1}$ are both complex numbers) such that $\boldsymbol{A} * \boldsymbol{X}=\lambda * \boldsymbol{X}$, then $\lambda$ is said to be the left eigenvalue of $\boldsymbol{A}$, and $\boldsymbol{X}$ is the left eigenvector corresponding to $\lambda$.

For the sake of distinction, we call the left eigenvalue and the left eigenvector under Definition 4.1 the left quaternion eigenvalue and the left quaternion eigenvector respectively.

According to the new definition of quaternion matrix multiplication and $\boldsymbol{A} * \boldsymbol{X}=\lambda * \boldsymbol{X}$, we can derive that $(\lambda * \tilde{\boldsymbol{E}}-\boldsymbol{A}) * \boldsymbol{X}=\mathbf{0}$. Thus $f(\lambda)=\|\lambda * \tilde{\boldsymbol{E}}-\boldsymbol{A}\|$ is said to be the characteristic polynomial of $\boldsymbol{A}$ (where the operator $\|\cdot\|$ denotes the determinant of quaternion matrix under Definition 3.4).

Theorem 4.1. A $n \times n$ quaternion matrix $A=A_{0}+A_{1} j$ (where $A_{0}, A_{1}$ both are complex matrices), if $\lambda$ and $\mu$ are the left eigenvalues of $A_{0}$ and $A_{1}$ respectively, then $\lambda+a j$ and $b+\mu j \quad(\forall a \in C$, $\forall b \in C$ ) are the left quaternion eigenvalues of $\boldsymbol{A}$.

Proof. Since $\lambda$ and $\mu$ are the left eigenvalues of $\boldsymbol{A}_{0}$ and $\boldsymbol{A}_{1}$ respectively, then there exist nonzero vectors 
$\boldsymbol{\xi} \in \boldsymbol{C}^{n \times 1}$ and $\boldsymbol{\eta} \in \boldsymbol{C}^{n \times 1}$ such that $\boldsymbol{A}_{0} \boldsymbol{\xi}=\lambda \boldsymbol{\xi}, \boldsymbol{A}_{1} \boldsymbol{\eta}=\mu \boldsymbol{\eta}$. We have

$$
\boldsymbol{A} * \boldsymbol{\xi}=\left(\boldsymbol{A}_{0}+\boldsymbol{A}_{1} j\right) *(\boldsymbol{\xi}+\mathbf{0} j)=\boldsymbol{A}_{0} \boldsymbol{\xi}=\lambda \boldsymbol{\xi}=(\lambda+a j) * \boldsymbol{\xi},
$$

for $\forall a \in C$.

$$
\begin{aligned}
\boldsymbol{A} *(\boldsymbol{\eta} j) & =\left(\boldsymbol{A}_{0}+\boldsymbol{A}_{1} j\right) *(\mathbf{0}+\boldsymbol{\eta} j)=\boldsymbol{A}_{1} \boldsymbol{\eta} j=\mu \boldsymbol{\eta} j \\
& =(b+\mu j) *(\boldsymbol{\eta} j)
\end{aligned}
$$

for $\forall b \in C$.

So $\lambda+a j$ and $b+\mu j$ are all the left quaternion eigenvalues of $\boldsymbol{A}$.

The proof is complete.

Similarly, we introduce a new right quaternion eigenvalue concept.

Definition 4.2. For any matrix $\boldsymbol{A}=\left(a_{i j}\right) \in \boldsymbol{H}^{n \times n}$, if there exists nonzero quaternion vector $\in \boldsymbol{H}^{1 \times n}$ and quaternion $\mu=\mu_{0}+\mu_{1} j$ (where $\mu_{0}, \mu_{1}$ are both complex numbers) such that

$\boldsymbol{Y} * \boldsymbol{A}=\mu * \boldsymbol{Y}$, then $\mu$ is said to be the right quaternion eigenvalue of $\boldsymbol{A}$, and $\boldsymbol{Y}$ is the right quaternion eigenvector corresponding to $\mu$.

For the right eigenvalue of quaternion matrix, we have the following theorem:

Theorem 4.2. $A \quad n \times n$ quaternion matrix $A=A_{0}+A_{1} j$ (where $A_{0}, A_{1}$ are both complex matrices), if $\lambda$ and $\mu$ are the right eigenvalues of $\boldsymbol{A}_{0}$ an $\boldsymbol{A}_{1}$ respectively, then $\lambda+a j$ and $b+\mu j(\forall a \in C, \forall b \in C)$ are the right quaternion eigenvalues of $\boldsymbol{A}$.

Proof. Since $\lambda$ and $\mu$ are the right eigenvalues of $\boldsymbol{A}_{0}$ and $\boldsymbol{A}_{1}$ respectively, then there exist nonzero vectors $\xi \in C^{1 \times n}$ and $\boldsymbol{\eta} \in C^{1 \times n}$ such that $\xi A_{0}=\lambda \xi, \eta A_{1}=\mu \eta$. We have

$$
\begin{gathered}
\boldsymbol{\xi} * \boldsymbol{A}=(\boldsymbol{\xi}+\mathbf{0} j) *\left(\boldsymbol{A}_{0}+\boldsymbol{A}_{1} j\right)=\boldsymbol{\xi} \boldsymbol{A}_{0} \\
=\lambda \boldsymbol{\xi}=(\lambda+a j) * \boldsymbol{\xi}, \text { for } \forall a \in C \\
(\boldsymbol{\eta} j) * \boldsymbol{A}=(\mathbf{0}+\boldsymbol{\eta} j) *\left(\boldsymbol{A}_{0}+\boldsymbol{A}_{1} j\right)=\boldsymbol{\eta} \boldsymbol{A}_{1} j=\mu \boldsymbol{\eta} j \\
=(b+\mu j) *(\boldsymbol{\eta} j), \text { for } \forall b \in C
\end{gathered}
$$

So $\lambda+a j$ and $b+\mu j$ are the right quaternion eigenvalues of $\boldsymbol{A}$.

The proof is complete.

Theorem 4.3. If the left eigenvalues of $A_{0}$ are $\lambda_{1}, \lambda_{2}, \cdots, \lambda_{k}$ and the left eigenvalues of $\boldsymbol{A}_{1}$ are $\mu_{1}, \mu_{2}, \cdots, \mu_{m}$ (where $\boldsymbol{A}_{0}, \boldsymbol{A}_{1}$ both are complex matrices), then the left quaternion eigenvalues of matrix $\boldsymbol{A}=\boldsymbol{A}_{0}+\boldsymbol{A}_{1} j$ are $\left\{\lambda_{\mathrm{s}}+a j\right.$ or $\left.b+\mu_{t} j, \forall a \in C, \forall b \in C, s=1, \cdots, k, t=1, \cdots, m\right\}$.

Proof. Suppose that $\gamma$ is arbitrary left quaternion eigenvalue of $\boldsymbol{A}$, then $\exists \boldsymbol{\psi} \neq \mathbf{0}, \boldsymbol{\psi}=\boldsymbol{\psi}_{0}+\boldsymbol{\psi}_{1} j \in \boldsymbol{H}^{n \times 1}$, such that $A * \psi=\gamma * \psi$, that is, $\left\{\begin{array}{c}A_{0} \psi_{0}=\gamma_{0} \psi_{0} \\ A_{1} \psi_{1}=\gamma_{1} \psi_{1}\end{array}\right.$. Since $\boldsymbol{\psi} \neq \mathbf{0}$, we know that both $\boldsymbol{\psi}_{0}$ and $\boldsymbol{\psi}_{1}$ are not zeroes. So there are two cases as follows:
1) When $\boldsymbol{\psi} \neq \mathbf{0}$, obviously, we have $\gamma_{0} \in\left\{\lambda_{1}, \lambda_{2}, \cdots, \lambda_{k}\right\}$.

So, $\gamma \in\left\{\lambda_{i}+a j, i=1,2, \cdots, k\right\}$.

2) When $\boldsymbol{\psi} \neq \mathbf{0}$, obviously, we have $\gamma_{1} \in\left\{\mu_{1}, \mu_{2}, \cdots, \mu_{m}\right\}$.

So, $\gamma \in\left\{b+\mu_{t} j, t=1,2, \cdots, m\right\}$.

To sum up 1), 2) and Theorem 4.1, we can draw the conclusion.

The proof is complete.

Theorem 4.4. If the right eigenvalues of $A_{0}$ are $\lambda_{1}, \lambda_{2}, \cdots, \lambda_{k}$ and the right eigenvalues of $A_{1}$ are $\mu_{1}, \mu_{2}, \cdots, \mu_{m}$ (where $\boldsymbol{A}_{0}, \boldsymbol{A}_{1}$ both are complex matrices), then the right quaternion eigenvalues of matrix $\boldsymbol{A}=\boldsymbol{A}_{0}+\boldsymbol{A}_{1} j$ are $\left\{\lambda_{\mathrm{s}}+a j\right.$ or $\left.b+\mu_{t}, \forall a \in C, \forall b \in C, s=1, \cdots, k, t=1, \cdots, m\right\}$.

This proof is similar toTheorem 4.3. So we omit it here.

Theorem 4.5. Let $\boldsymbol{A} \in \boldsymbol{H}^{n \times n}$, then $\boldsymbol{A}$ and $\boldsymbol{A}^{T}$ have the same quaternion left (right) eigenvalues.

Proof. Since $\boldsymbol{A}=\boldsymbol{A}_{0}+\boldsymbol{A}_{1} j$ (where $\left.\boldsymbol{A}_{0} \in \boldsymbol{C}^{n \times n}, \boldsymbol{A}_{1} \in C^{n \times n}\right)$, then $\boldsymbol{A}^{T}=\boldsymbol{A}_{0}^{T}+\boldsymbol{A}_{1}^{T} \boldsymbol{j}$. We know $A_{i}$ and $A_{i}^{T}$ have the same left (right) eigenvalues ( $i=1,2$ ). By Theorem 4.3 and Theorem 4.4, we can draw the conclusion.

The proof is complete.

Theorem 4.6. Let $\boldsymbol{A} \in \boldsymbol{H}^{n \times n}$ and $\mu, \lambda \in H$ be given. If $\lambda(\mu)$ is the left (right) quaternion eigenvalue of $\boldsymbol{A}$, then $\lambda(\mu)$ is the right (left) quaternion eigenvalue of $\boldsymbol{A}$.

Proof. Since $\lambda$ is the left quaternion eigenvalue of $\boldsymbol{A}$, then there exits nonzero vector $\boldsymbol{\xi}$ such that $\boldsymbol{A} * \boldsymbol{\xi}=\lambda * \boldsymbol{\xi}$. Then $(\boldsymbol{A} * \boldsymbol{\xi})^{T}=(\lambda * \boldsymbol{\xi})^{T}$, we can have $\boldsymbol{\xi}^{T} * \boldsymbol{A}^{T}=\lambda * \boldsymbol{\xi}^{T}$. So $\lambda$ is the right quaternion eigenvalue of $\boldsymbol{A}^{T}$, by Theorem 4.5, we know $\lambda$ is the right quaternion eigenvalue of $\boldsymbol{A}$. The same proof to $\mu$.

So, the proof is complete.

Specially, when $\boldsymbol{A} \in \boldsymbol{C}^{n \times n}$, if $\lambda(\mu)$ is the left (right) eigenvalue of $\boldsymbol{A}$, then $\lambda(\mu)$ is the right (left) eigenvalue of $\boldsymbol{A}$.

Note: By the new definition of quaternion multiplication, the left quaternion eigenvalue of a quaternion matrix is equivalent to its right quaternion eigenvalue. So they are both called quaternion eigenvalue of the quaternion matrix.

In the following, we show an important result.

Theorem 4.7. Let $\boldsymbol{A}, \boldsymbol{B} \in \boldsymbol{H}^{n \times n}$ be given. If $\boldsymbol{A} \backsim \boldsymbol{B}$, then $\boldsymbol{A}$ and $\boldsymbol{B}$ have the same eigenvalues.

Proof. Since $\boldsymbol{A} \backsim \boldsymbol{B}$, there exists an invertible matrix $\boldsymbol{P} \in \boldsymbol{H}^{n \times n}$ such that $\boldsymbol{A}=\boldsymbol{P}^{-} * \boldsymbol{B} * \boldsymbol{P}$, that is equivalent to $\boldsymbol{A}=\boldsymbol{P}_{0}^{-1} \boldsymbol{B} \boldsymbol{P}_{0}$ and $\boldsymbol{A}_{1}=\boldsymbol{P}_{1}^{-1} \boldsymbol{B}_{1} \boldsymbol{P}_{1}$ (where $\boldsymbol{A}=\boldsymbol{A}_{0}+\boldsymbol{A}_{1} \boldsymbol{j}$, $\left.\boldsymbol{B}=\boldsymbol{B}_{0}+\boldsymbol{B}_{1} j, \quad \boldsymbol{P}=\boldsymbol{P}_{0}+\boldsymbol{P}_{1} j\right)$. We know $\boldsymbol{B}_{s}$ and $\boldsymbol{A}_{s}$ $(s=0,1)$ have the same eigenvalues. By Theorem 4.3 and Theorem 4.4, we can draw that $A$ and $B$ have 
the same eigenvalues.

The proof is complete.

Theorem 4.8 (The generalized Cayley-Hamilton theorem over quaternion division algebra). A quaternion matrix $\boldsymbol{A}$ must be the root of its characteristic polynomial $f(\lambda)=\|\lambda * \tilde{\boldsymbol{E}}-\boldsymbol{A}\|$.

Proof. According to Definition 3.4, we know that:

$$
\begin{aligned}
f(\lambda) & =f\left(\lambda_{0}+\lambda_{1} j\right)=\|\lambda * \tilde{\boldsymbol{E}}-\boldsymbol{A}\| \\
& =\left\|\left(\lambda_{0} \boldsymbol{E}+\lambda_{1} \boldsymbol{E} j\right)-\left(\boldsymbol{A}_{0}+\boldsymbol{A}_{1} j\right)\right\| \\
& =\left|\lambda_{0} \boldsymbol{E}-\boldsymbol{A}_{0}\right|+\left|\lambda_{1} \boldsymbol{E}-\boldsymbol{A}_{1}\right| j=g\left(\lambda_{0}\right)+h\left(\lambda_{1}\right) j
\end{aligned},
$$

where $g\left(\lambda_{0}\right)=\left|\lambda_{0} \boldsymbol{E}-\boldsymbol{A}_{0}\right|, h\left(\lambda_{1}\right)=\left|\lambda_{1} \boldsymbol{E}-\boldsymbol{A}_{1}\right|$.

According to the Cayley-Hamilton theorem on complex field, we know $g\left(\boldsymbol{A}_{0}\right)=\mathbf{0}, h\left(\boldsymbol{A}_{1}\right)=\mathbf{0}$. So, $f(\boldsymbol{A})=g\left(\boldsymbol{A}_{0}\right)+h\left(\boldsymbol{A}_{1}\right)=\mathbf{0}$. It indicates that quaternion matrix $A$ must be the root of its characteristic polynomial $f(\lambda)$.

So, the proof is complete.

Theorem 4.9. Let (where $\boldsymbol{A}=\boldsymbol{A}_{0}+\boldsymbol{A}_{1} \boldsymbol{j} \in \boldsymbol{H}^{n \times n}$ $\boldsymbol{A}_{0}, \boldsymbol{A}_{1} \in \boldsymbol{C}^{n \times n}$ ) be given. $\boldsymbol{A}$ is a diagonalizable matrix if and only if both $\boldsymbol{A}_{0}$ and $\boldsymbol{A}_{1}$ are diagonalizable matrices.

Proof. $\boldsymbol{A}$ is diagonalizable matrix , that is ,there exits an invertible quaternion matrix $\boldsymbol{P}$ such that

$\boldsymbol{A}=\boldsymbol{P}^{-} * \Delta * \boldsymbol{P}$. It is equivalent to $\boldsymbol{A}_{0}=\boldsymbol{P}_{0}^{-1} \Delta_{0} \boldsymbol{P}_{0}$ and $\boldsymbol{A}_{1}=\boldsymbol{P}_{1}^{-1} \Delta_{1} \boldsymbol{P}$ (where $\Delta=\Delta_{0}+\Delta_{1} j$ is diagonal matrix). So, $\boldsymbol{A}$ is diagonalizable matrix if and only if both $\boldsymbol{A}_{0}$ and $\boldsymbol{A}_{1}$ are diagonalizable matrices.

The proof is complete.

Corollary 4.9. Let $\boldsymbol{A}=\boldsymbol{A}_{0}+\boldsymbol{A}_{1} \boldsymbol{j} \in \boldsymbol{H}^{n \times n}$ (where $\boldsymbol{A}_{0}, \boldsymbol{A}_{1} \in \boldsymbol{C}^{n \times n}$ ) be given. If $\boldsymbol{A}_{0}$ and $\boldsymbol{A}_{1}$ both have $n$ different eigenvalues, then $\boldsymbol{A}$ is diagonalizable matrix.

Corollary 4.9'. Let $\boldsymbol{A}=\boldsymbol{A}_{0}+\boldsymbol{A}_{1} j \in \boldsymbol{H}^{n \times n}$ (where $\boldsymbol{A}_{0}, \boldsymbol{A}_{1} \in \boldsymbol{C}^{n \times n}$ ) be given. Quaternion matrix $\boldsymbol{A}$ is diagonalizable matrix if and only if and $\boldsymbol{A}_{1}$ both have $n$ linearly independent eigenvactors.

Corollary 4.9". Let $\boldsymbol{A}=\boldsymbol{A}_{0}+\boldsymbol{A}_{1} j \in \boldsymbol{H}^{n \times n}$ (where $\boldsymbol{A}_{0}, \boldsymbol{A}_{1} \in \boldsymbol{C}^{n \times n}$ ) be given. Quaternion matrix $\boldsymbol{A}$ is diagonalizable matrix if and only if the geometric multiplicity of $\boldsymbol{A}_{0}$ and $\boldsymbol{A}_{1}$ both equal their algebraic multiplicity respectively.

In Section 3, we have given the new definition of the inverse of quaternion matrix, but that of quaternion is not defined. In fact, a quaternion can be treated as a $1 \times 1$ matrix. So we can define the inverse of quaternion as follows:

Definition 4.3. For any quaternion $a=a_{0}+a_{1} j$, if neither of $a_{0}$ and $a_{1}$ are zeroes, then $a^{-}=a_{0}^{-1}+a_{1}^{-1} j$ is said to be the inverse of $a$, where $a_{s}^{-1}(s=0,1)$ is the reciprocal of $a_{s}$.

It is easy to verify the following facts. For any $a, b \in H$, we have:

1) $a_{u} * a=a * a_{u}=a$

2) $a+b=b+a$;

3) $(a+b) * c=a * c+b * c$;

4) $a^{n}=\left(a_{0}\right)^{n}+\left(a_{1}\right)^{n} j$;

5) If $a=a_{0}+a_{1} j$ has the inverse $a^{-}$, then $a * a^{-}=a_{u}$.

In addition, we discover that there are some special phenomena about the roots of quaternion polynomial under the new definition of quaternion multiplication.

Definition 4.4. The polynomial which has the form as follows: $a_{0} * x^{* 0}+a_{1} * x^{*(n-1)}+\cdots+a_{n-1} * x^{* 1}+a_{n} * x^{* 0}$ is said to be quaternion polynomial with complex coefficients (where $a_{i}, i=0,1, \cdots, n$ are all complex numbers, $x=x_{0}+x_{1} j, x^{* 0}$ is the *-product of $i$ quaternion $x$ and $x^{* 0}$ is unit quaternion).

Theorem 4.10. Let $f(x)$ be a quaternion polynomial with complex coefficients. Then $f(x)$ has infinite quaternion roots.

Proof. By Fundamental Theorem of algebra, $f(x)$ exists at least one complex root $x_{0}$, then for any given complex number $x_{1}$, obviously, $x_{0}+x_{1} j$ is the root of $f(x)$.

The proof is complete.

Theorem 4.11. Let $f(x)$ be a quaternion polynomial with complex coefficients and $\boldsymbol{A}=\boldsymbol{A}_{0}+\boldsymbol{A}_{1} j \in \boldsymbol{H}^{n \times n}$ be a given quaternion matrix (where, both $\boldsymbol{A}_{0}$ and $\boldsymbol{A}_{1}$ are compex matrices). If $\lambda$ is the eigenvalue of $\boldsymbol{A}_{0}$, then $f(\lambda)$ is the eigenvalue of $f(\boldsymbol{A})$.

Proof. According to the new definition of quaternion multiplication, we can easily obtain $f(\boldsymbol{A})=f\left(\boldsymbol{A}_{0}\right)$. Since $\lambda$ is the eigenvalue of $\boldsymbol{A}_{0}$, so $f(\lambda)$ is the eigenvalue of $f\left(\boldsymbol{A}_{0}\right)$.

The proof is complete.

Under the new concept system, we can also solve the problems of existence and uniqueness of the solutions to the quaternion system of linear equations $\boldsymbol{A} * \boldsymbol{X}=\boldsymbol{b}$, where operator ' $*$ ' denotes the new multiplication of quaternion matrices.

As we known, for any $\boldsymbol{A} \in \boldsymbol{H}^{m \times n}, \boldsymbol{A}$ can be represented uniquely as $\boldsymbol{A}=\boldsymbol{A}_{0}+\boldsymbol{A}_{1} j$, where $\boldsymbol{A}_{s} \quad(s=0,1)$ are $n \times n$ complex matrices. Let

$\boldsymbol{X}=\left(x_{10}+x_{11} j, x_{20}+x_{21} j, \cdots, x_{n 0}+x_{n 1} j\right)^{\mathrm{T}}$ and $\boldsymbol{b}=\left(b_{10}+b_{11} j, b_{20}+b_{21} j, \cdots, b_{n 0}+b_{n 1} j\right)^{\mathrm{T}}$ be $n \times 1$ quaternion vectors, then the following theorems are valid.

Theorem 4.12. Let $\boldsymbol{A}=\boldsymbol{A}_{0}+\boldsymbol{A}_{1} \boldsymbol{j} \in \boldsymbol{H}^{n \times n}$ be given and $\boldsymbol{X}=\boldsymbol{X}_{0}+\boldsymbol{X}_{1} j$ be $n \times 1$ quaternion vector. If rank $\left(\boldsymbol{A}_{s}\right)=r_{s}$ and the fundamental system of solutions to the system of homogeneous linear equations $\boldsymbol{A}_{s} \boldsymbol{X}_{s}=\mathbf{0}$ is $\eta_{i 1}, \eta_{i 2}, \cdots, \eta_{i\left(n-r_{i}\right)} \quad(s=0,1)$ respectively, then any solution to the quaternion system of homogeneous linear 
equations $\boldsymbol{A} * \boldsymbol{X}=\mathbf{0}$ can be expressed as follows:

$$
\begin{aligned}
\boldsymbol{X}= & \left(c_{01} \eta_{01}+c_{02} \eta_{02}+\cdots+c_{0\left(n-r_{0}\right)} \eta_{0\left(n-r_{0}\right)}\right), \\
& +\left(c_{11} \eta_{11}+c_{12} \eta_{12}+\cdots c_{1\left(n-r_{1}\right)} \eta_{1\left(n-r_{1}\right)}\right) j
\end{aligned}
$$

where $c_{s t_{s}} \in C, t_{s}=0, \cdots, n-r_{s}, s=0,1$.

Proof. By the new definition of quaternion matrix multiplication, the quaternion system of homogeneous linear equations $\boldsymbol{A} * \boldsymbol{X}=\mathbf{0}$ is equivalent to the system of homogeneous linear equations $\left\{\begin{array}{l}\boldsymbol{A}_{0} \boldsymbol{X}_{0}=\mathbf{0} \\ \boldsymbol{A}_{1} \boldsymbol{X}_{1}=\mathbf{0}\end{array}\right.$. Since any solution to the system of homogeneous linear equations $\boldsymbol{A}_{s} \boldsymbol{X}_{s}=\mathbf{0}$ can expressed as

$$
\begin{aligned}
& \boldsymbol{X}_{s}=\left(c_{s 1} \eta_{s 1}+c_{s 2} \eta_{s 2}+\cdots+c_{s\left(n-r_{0}\right)} \eta_{s\left(n-r_{0}\right)}\right) \\
& \text { (where } \left.c_{s t_{s}} \in C, t_{s}=1, \cdots, n-r_{s}, s=0,1\right)
\end{aligned}
$$

and the solutions of the quaternion system of homogeneous linear equations $\boldsymbol{A} * \boldsymbol{X}=\mathbf{0}$ are $\boldsymbol{X}=\boldsymbol{X}_{0}+\boldsymbol{X}_{1} j$. So we can draw the conclusion.

So, the proof is complete.

Corollary 4.12. Let $A=A_{0}+A_{1} j$ be a given quaternion matrix (where $A_{s} \in C^{m \times n}, s=0,1$ ).

If rank $\left(A_{0}\right)=\operatorname{rank}\left(A_{1}\right)=n$, then the quaternion system of homogeneous linear equations $\boldsymbol{A} * \boldsymbol{X}=\mathbf{0}$ has unique solution $\boldsymbol{X}=\mathbf{0}=\{0,0, \cdots 0\}^{\mathrm{T}}$.

Corollary 4.12'. Let $\boldsymbol{A}=\boldsymbol{A}_{0}+\boldsymbol{A}_{1} j$ be a given quaternion matrix (where $\boldsymbol{A}_{s} \in \boldsymbol{C}^{m \times n}, s=0,1$ ). If rank $\left(A_{0}\right)<n$ and rank $\left(A_{1}\right)=n$, then the quaternion system of homogeneous linear equations $\boldsymbol{A} * \boldsymbol{X}=\mathbf{0}$ only exists complex solutions.

Theorem 4.13. Let $\boldsymbol{A}=\boldsymbol{A}_{0}+\boldsymbol{A}_{1} j$ be a given quaternion matrix, $\boldsymbol{X}=\boldsymbol{X}_{0}+\boldsymbol{X}_{1} j$ and $\boldsymbol{b}=\boldsymbol{b}_{0}+\boldsymbol{b}_{1} j$ be quaternion vectors (where $\boldsymbol{A}_{s} \in \boldsymbol{C}^{m \times n}, \boldsymbol{X}_{s} \in \boldsymbol{C}^{n \times 1}$, $\left.\boldsymbol{b}_{s}=\left(b_{s 1}, b_{s 2}, \cdots, b_{s n}\right)^{\mathrm{T}}, \quad b_{s t} \in C, \quad s=0,1, \quad t=1,2, \cdots, n\right)$. If there is at least one $s_{0} \in\{0,1\}$ such that rank $\left(A_{s_{0}}\right)$ $<\operatorname{rank}\left(A_{s_{0}} \vdots b_{s_{0}}\right)$, then the quaternion system of linear equations $\boldsymbol{A} * \boldsymbol{X}=\boldsymbol{b}$ has no solution.

Proof. By the new definition of quaternion matrix multiplication, the quaternion system of linear equations $\boldsymbol{A} * \boldsymbol{X}=\boldsymbol{b}$ is equivalent to the system of linear equations $\left\{\begin{array}{l}\boldsymbol{A}_{0} \boldsymbol{X}_{0}=\boldsymbol{b}_{0} \\ \boldsymbol{A}_{1} \boldsymbol{X}_{1}=\boldsymbol{b}_{1}\end{array}\right.$, since $\operatorname{rank}\left(A_{s_{0}}\right)<\operatorname{rank}\left(A_{s_{0}} \vdots b_{s_{0}}\right)$, the system of linear equations $\left\{\begin{array}{l}\boldsymbol{A}_{0} \boldsymbol{X}_{0}=\boldsymbol{b}_{0} \\ \boldsymbol{A}_{1} \boldsymbol{X}_{1}=\boldsymbol{b}_{1}\end{array}\right.$ have no solution, that is, the quaternion system of linear equations $\boldsymbol{A} * \boldsymbol{X}=\boldsymbol{b}$ has no solution.

So, the proof is complete.

Theorem 4.14. Let $\boldsymbol{A}=\boldsymbol{A}_{0}+\boldsymbol{A}_{1} j$ be a given quaternion matrix and $\boldsymbol{X}=\boldsymbol{X}_{0}+\boldsymbol{X}_{1} j$ be a given quaternion vector (where $\boldsymbol{A}_{s} \in \boldsymbol{C}^{m \times n}, \boldsymbol{X}_{s} \in \boldsymbol{C}^{n \times 1}, \quad s=0,1$ ). We suppose that the fundamental system of solutions to the system of linear equations $\boldsymbol{A}_{s} \boldsymbol{X}_{s}=\mathbf{0}$ is $\eta_{s 1,} \eta_{s 2}, \cdots, \eta_{s\left(n-r_{s}\right)}$ $(s=0,1)$ respectively and $\xi_{s}(s=0,1)$ is a special solution of the system of linear equations $A_{s} X_{s}=b_{s}$ respectively, and $\operatorname{rank}\left(\boldsymbol{A}_{s}\right)=\operatorname{rank}\left(\boldsymbol{A}_{s} \vdots \boldsymbol{b}_{s}\right) \quad(s=0,1)$, then any solution to the quaternion system of linear equations $\boldsymbol{A} * \boldsymbol{X}=\boldsymbol{b}$ can be expressed as:

$$
\begin{aligned}
\boldsymbol{X}= & \left(\xi_{0}+c_{01} \eta_{01}+c_{02} \eta_{02}+\cdots+c_{0\left(n-r_{0}\right)} \eta_{0\left(n-r_{0}\right)}\right) \\
& +\left(\xi_{1}+c_{11} \eta_{11}+c_{12} \eta_{12}+\cdots c_{1\left(n-r_{1}\right)} \eta_{1\left(n-r_{1}\right)}\right) j
\end{aligned}
$$

Proof. By the new definition of quaternion matrix multiplication, the quaternion system of linear equations $\boldsymbol{A} * \boldsymbol{X}=\boldsymbol{b}$ is equivalent to the system of linear equations $\left\{\begin{array}{l}\boldsymbol{A}_{0} \boldsymbol{X}_{0}=\boldsymbol{b}_{0} \\ \boldsymbol{A}_{1} \boldsymbol{X}_{1}=\boldsymbol{b}_{1}\end{array}\right.$. Since any solution to the system of linear equations $\boldsymbol{A}_{s} \boldsymbol{X}_{s}=\boldsymbol{b}_{s}$ can expressed as follows:

$$
\boldsymbol{X}_{s}=\xi_{s}+c_{s 1} \eta_{s 1}+c_{s 2} \eta_{s 2}+\cdots+c_{s\left(n-r_{s}\right)} \eta_{s\left(n-r_{s}\right)} \quad \text { (where }
$$

$\left.c_{s t} \in C, t=1,2, \cdots, n-r_{s}, s=0,1\right)$, so any solution to the system of quaternion linear equations $\boldsymbol{A} * \boldsymbol{X}=\boldsymbol{b}$ can be expressed as:

$$
\begin{aligned}
\boldsymbol{X}= & \left(\xi_{0}+c_{01} \eta_{01}+c_{02} \eta_{02}+\cdots+c_{0\left(n-r_{0}\right)} \eta_{0\left(n-r_{0}\right)}\right) \\
& +\left(\xi_{1}+c_{11} \eta_{11}+c_{12} \eta_{12}+\cdots c_{1\left(n-r_{1}\right)} \eta_{1\left(n-r_{1}\right)}\right) j
\end{aligned} .
$$

The proof is complete.

Theorem 4.15. Let $\boldsymbol{A}=\boldsymbol{A}_{0}+\boldsymbol{A}_{1} j$ be a given quaternion matrix, $\boldsymbol{X}=\boldsymbol{X}_{0}+\boldsymbol{X}_{1} j$ and $\boldsymbol{b}=\boldsymbol{b}_{0}+\boldsymbol{b}_{1} j$ be quaternion vectors (where $\boldsymbol{A}_{s} \in \boldsymbol{C}^{m \times n}, \boldsymbol{X}_{s} \in \boldsymbol{C}^{n \times 1}, \boldsymbol{b}_{s} \in C^{n \times 1}$, $(s=0,1))$. If $\operatorname{rank}\left(\boldsymbol{A}_{s}\right)=\operatorname{rank}\left(\boldsymbol{A}_{s} \vdots \boldsymbol{b}_{s}\right)=n \quad(s=0,1)$, then the quaternion system of linear equations $\boldsymbol{A} * \boldsymbol{X}=\boldsymbol{b}$ exists unique solution.

Proof. By the new definition of quaternion matrix multiplication, the quaternion system of linear equations $A * X=b$ is equivalent to the system of linear equations $\left\{\begin{array}{c}\boldsymbol{A}_{0} \boldsymbol{X}_{0}=\boldsymbol{b}_{0} \\ \boldsymbol{A}_{1} \boldsymbol{X}_{1}=\boldsymbol{b}_{1}\end{array}\right.$, and $\operatorname{rank}\left(\boldsymbol{A}_{s}\right)=\operatorname{rank}\left(\boldsymbol{A}_{s} \boldsymbol{b}_{s}\right)=n$, we know the system of linear equations $\left\{\begin{array}{l}\boldsymbol{A}_{0} \boldsymbol{X}_{0}=\boldsymbol{b}_{0} \\ \boldsymbol{A}_{1} \boldsymbol{X}_{1}=\boldsymbol{b}_{1}\end{array}\right.$ have unique solution. So the quaternion system of linear equations $\boldsymbol{A} * \boldsymbol{X}=\boldsymbol{b}$ exists unique solution.

The proof is complete.

Corollary 4.15. Let $\boldsymbol{A}=\boldsymbol{A}_{0}+\boldsymbol{A}_{1} j$ be a given $n \times n$ quaternion matrix and $\boldsymbol{b}=\boldsymbol{b}_{0}+\boldsymbol{b}_{1} j$ be a given $n \times 1$ quaternion vector. If $\operatorname{rank}\left(\boldsymbol{A}_{s}\right)=\operatorname{rank}\left(\boldsymbol{A}_{s} \vdots \boldsymbol{b}_{s}\right)=n$ $(s=0,1)$, then the solution of the quaternion system of equations $\boldsymbol{A} * \boldsymbol{X}=\boldsymbol{b}$ is $\boldsymbol{X}=\boldsymbol{A}^{-1} * \boldsymbol{b}$.

Corollary 4.15'. Let $\boldsymbol{A} \in \boldsymbol{C}^{m \times n}$ and $\boldsymbol{b}=\boldsymbol{b}_{0}+\boldsymbol{b}_{1} j$ (where $\boldsymbol{b}_{s} \in \boldsymbol{C}^{n \times 1}, s=0,1, \boldsymbol{b}_{1} \neq \mathbf{0}$ ) be given. Then the 
quaternion system of linear equations $\boldsymbol{A} * \boldsymbol{X}=\boldsymbol{b}$ has no solution.

Corollary 4.15". Let $\boldsymbol{A} \in \boldsymbol{C}^{m \times n}$ and $\boldsymbol{b} \in \boldsymbol{C}^{n \times 1}$ be given. If $\operatorname{rank}(\boldsymbol{A})=\operatorname{rank}(\boldsymbol{A} \vdots \boldsymbol{b})$, then any solution to the quaternion system of linear equations $\boldsymbol{A} * \boldsymbol{X}=\boldsymbol{b}$ can expressed as $\boldsymbol{X}=\boldsymbol{A}^{-1} \boldsymbol{b}+a j$, where $a \in C$.

\section{References}

[1] L. X. Chen, "Inverse Matrix and Properties of Double Determinant over Quaternion TH Field," Science in China (Series A), Vol. 34, No. 5, 1991, pp. 25-35.

[2] L. X. Chen, "Generalization of Cayley-Hamilton Theorem over Quaternion Field," Chinese Science Bulletin, Vol. 17, No. 6, 1991, pp. 1291-1293.

[3] R, M. Hou, X. Q. Zhao and 1. T. Wang, "The Double Determinant of Vandermonde's Type over Quaternion Field," Applied Mathematics and Mechanics, Vol. 20, No. 9, 1999, pp. 100-107.

[4] L. P. Huang, "The Determinants of Quateruion Matrices and Their Propoties," Journal of Mathematics Study, Vol. 2, 1995, pp. 1-13.

[5] J. L. Wu, L. M. Zou, X. P. Chen and S. J. Li, "The Estimation of Eigenvalues of Sum, Difference, and Tensor Product of Matrices over Quaternion Division Algebra," Linear Algebra and its Applications, Vol. 428, 2008, pp. 3023-3033. doi:10.1016/j.laa.2008.02.008

[6] T. S. Li, "Properties of Double Determinant over Quaternion Field," Journal of Central China Normal University, Vol. 1, 1995, 3-7. doi:10.1007/BF02652076

[7] B. X. Tu, "Dieudonne Determinants of Matrices over a Division Ring," Journal of Fudan university, 1990A, Vol. 1, pp. 131-138.

[8] B. X. Tu, "Weak Direct Products and Weak Circular Product of Matrices over the Real Quaternion Division Ring,” Journal of Fudan University, Vol. 3, 1991, p. 337.

[9] J. L. Wu, "Distribution and Estimation for Eigenvalues of Real Quaternion Matrices," Computers and Mathematics with Applications, Vol. 55, 2008, pp. 1998-2004. doi:10.1016/i.camwa.2007.07.013

[10] B. J. Xie, "Theorem and Application of Determinants Spread out of Self-Conjugated Matrix," Acta Mathematica Sinica, Vol. 5, 1980, pp. 678-683.

[11] Q. C. Zhang, "Properties of Double Determinant over the Quaternion Field and Its Applications," Acta Mathematica Sinica, Vol. 38, No. 2, 1995, pp. 253-259.

[12] W. J. Zhuang, "Inequalities of Eigenvalues and Singular Values for Quaternion Matrices," Advances in Mathematics, Vol. 4, 1988, pp. 403-406.

[13] W. Boehm, "On Cubics: A Survey, Computer Graphics and Image Processing," Vol. 19, 1982, pp. 201-226. doi:10.1016/0146-664X(82)90009-0

[14] G. Farin, "Curves and Surfaces for Computer Aided Geometric Design,” Academic Press, Inc., San Diego CA,
1990.

[15] K. Shoemake, “Animating Rotation with Quaternion Calculus," ACM SIGGRAPH, 1987, Course Notes, Computer Animation: 3-D Motion, Specification, and Control.

[16] Q. G. Wang, "Quaternion Transformation and Its Application to the Displacement Analysis of Spatial Mechanisms, Acta Mathematica Sinica, Vol. 15, No. 1, 1983, pp. 54-61.

[17] G. S. Zhang, "Commutativity of Composition for Finite Rotation of a Rigid Body," Acta Mechanica Sinica, Vol. 4, 1982.

[18] E. T. Browne, "The Characteristic Roots of a Matrix," Bulletin of the American Mathematical Society, Vol. 36, 1930, pp. 705-710. doi:10.1090/S0002-9904-1930-05041-7

[19] J. L. Wu and Y. Wang, "A New Representation Theory and Some Methods on Quaternion Division Algebra," Journal of Algebra, Vol. 14, No. 2, 2009, pp. 121-140.

[20] Q. W. Wang, "The General Solution to a System of Real Quaternion Matrix Equation," Computer and Mathematics with Applications, Vol. 49, 2005, pp. 665-675. doi:10.1016/j.camwa.2004.12.002

[21] G. B. Price, "An Introduction to Multicomplex Spaces and Functions," Marcel Dekker, New York, 1991.

[22] D. Rochon, “A Bicomplex Riemann Zeta Function," Tokyo Journal of Mathematics, Vol. 27, No. 2, 2004, pp. 357-369.

[23] S. P. Goyal and G. Ritu, "The Bicomplex Hurwitz Zeta function," The South East Asian Journal of Mathematics and Mathematical Sciences, 2006.

[24] S. P. Goyal, T. Mathur and G. Ritu, "Bicomplex Gamma and Beta Function," Journal of Raj. Academy Physical Sciences, Vol. 5, No. 1, 2006, pp. 131-142.

[25] J. N. Fan, "Determinants and Multiplicative Functionals on Quaternion Matrices," Linear Algebra and Its Applications, Vol. 369, 2003, pp. 193-201. doi:10.1016/S0024-3795(02)00722-X

[26] Q. W. Wang, "A System of Four Matrix Equations over Von Neumann Regular Rings and It Applications," Acta Mathematica Sinica, Vol. 21, 2005, pp. 323-334. doi:10.1007/s10114-004-0493-1

[27] Q. W. Wang, "A System of Matrix Equation and a Linear Matrix Equation over Arbitrary Regular Rings with Identity," Applied Linear Algebra, Vol. 384, 2004, pp. 43-54. doi:10.1016/j.laa.2003.12.039

[28] W. J. Zhuang, "The Guide of Matrix Theory over Quaternion Field," Science Press, Beijing, 2006, pp. 1-50.

[29] W. L. LI, "Quaternion Matrices," "National Defense Science and Technology University," Vol. 6, 2002, pp. 73-74.

[30] R. X. Jiang, "Linear Algebra," People's Educational Press, China, 1979, pp. 41-42. 